\title{
Socioeconomic, demographic and landscape factors associated with cutaneous leishmaniasis in Kurunegala District, Sri Lanka
}

Tharaka Wijerathna' ${ }^{1}$ Nayana Gunathilaka ${ }^{1 *}$, Kithsiri Gunawardena' ${ }^{1}$ and Wasana Rodrigo ${ }^{2}$

\begin{abstract}
Background: Leishmaniasis is a neglected tropical disease that affects countries in the developing world. In Sri Lanka, cutaneous leishmaniasis is the most common form of the disease. It is prevalent in dry and intermediate zones, mostly associated with rural settings. Understanding basic risk factors is critical in the management of the disease with effective interventions. This study is focused on assessing the demographic, socioeconomic and landscape factors associated with leishmaniasis in Kurunegala District, Sri Lanka.

Methods: A descriptive cross-sectional study was conducted. Households of the past patients and randomly selected households, which had no history of leishmaniasis cases were interviewed. The clinical, socioeconomic, demographic, landscape and awareness-related data were obtained using a pre-tested, interviewer-administered questionnaire.

Results: A total of 101 patients and a similar number of controls were included in the study. All the patients had the cutaneous form of the disease. Housewives and personnel with monthly incomes less than Rs. 10,000 (56.76 USD) were 3.9- and 9.5-times more prone to the disease, respectively, according to multivariate analysis. Presence of decaying garbage, termite hills, unclear areas, wet soil and gardening areas were always associated with the increased odds of acquiring the disease.

Conclusions: Demographic factors do not play a pivotal role in the prevalence of leishmaniasis in the area. Housewives, inhabitants with low incomes and individuals who live in areas with conditions suitable for sand fly breeding and resting are major groups with a higher risk of infection. Special attention must be given in raising awareness and environmental management in control activities.
\end{abstract}

Keywords: Leishmaniasis, Sand fly, Risk factors, Socioeconomic

\section{Background}

Leishmaniasis is a neglected tropical disease that mostly affects countries in the developing world [1]. Cutaneous leishmaniasis $(\mathrm{CL})$ is among the main clinical manifestations of this disease with $0.6-1.0$ million cases per year worldwide [1]. In Sri Lanka, CL is the most common type

*Correspondence: n.gunathilaka@kln.ac.lk

${ }^{1}$ Department of Parasitology, Faculty of Medicine, University of Kelaniya, Ragama, Sri Lanka

Full list of author information is available at the end of the article of leishmaniasis. Most patients have been recorded from dry and intermediate zones (transition zone between the dry and wet zones) $[2,3]$ until an outbreak was observed from the wet zone in 2018 [4].

In Sri Lanka, leishmaniasis is caused by Leishmania donovani zymodeme MON-37 [5] and transmitted by Phlebotomus argentipes [6]. Phlebotomus argentipes prefers areas with wet and humid soil enriched with decaying organic matter as breeding sites [7]. Dark and disorganized areas such as storerooms, piles of reed mats and storage areas of old and broken material are the

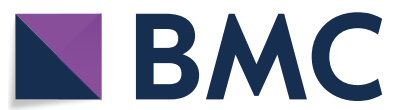

(c) The Author(s) 2020. This article is licensed under a Creative Commons Attribution 4.0 International License, which permits use, sharing, adaptation, distribution and reproduction in any medium or format, as long as you give appropriate credit to the original author(s) and the source, provide a link to the Creative Commons licence, and indicate if changes were made. The images or other third party material in this article are included in the article's Creative Commons licence, unless indicated otherwise in a credit line to the material. If material is not included in the article's Creative Commons licence and your intended use is not permitted by statutory regulation or exceeds the permitted use, you will need to obtain permission directly from the copyright holder. To view a copy of this licence, visit http://creativeco mmons.org/licenses/by/4.0/. The Creative Commons Public Domain Dedication waiver (http://creativecommons.org/publicdomain/ zero/1.0/) applies to the data made available in this article, unless otherwise stated in a credit line to the data. 
main potential indoor diurnal resting places of sand flies in these areas [8]. The betel and pepper cultivated fields and the inner side of termite mounds can be considered as main outdoor diurnal resting places [8]. Leishmaniasis in Sri Lanka, which is caused by $L$. donovani is most likely to be an anthroponosis. However, few published studies indicate the possibility of dogs being a reservoir host although there is no sufficient evidence $[9,10]$.

Risk factors of leishmaniasis include poor household characteristics, low level of education, lack of cleanliness in the surrounding environment and poor awareness about the disease [11-14]. The housing conditions such as cracked walls, dark humid corners, damp floors, and mud-plastered walls, which permit the easy entry, resting and breeding of sand flies, are known to increases the risk of infection of the inhabitants [14-17]. Furthermore, poor awareness of vectors, vector behaviour and protective measures of the disease, which is partly a result of the poor level of education, are also associated with a high risk of acquiring the infection [11-14]. Poor sanitization and garbage collection in the surroundings, which result in an environment preferable for sand fly survival, are also reported as risk factors for leishmaniasis $[11,18,19]$. Gender inequality is often encountered concerning leishmaniasis. Most often males are more prone to the disease than females [20-22]. However, this is not consistent across studies. Some studies suggest similar effects for both sexes while some studies indicate a higher susceptibility of females [23, 24]. Children usually have a lower risk than adults to acquire the infection [25, 26]; however, this is also not consistent for all settings. Other studies suggest that children have a higher risk [27].

Some associated factors are limited to a specific form of the disease [21-24, 28]. Further, these risk factors may differ from one geographical region to another [21, 22, $24,28]$. Therefore, demography, epidemiology of the disease and risk factors at endemic setting should be studied to determine proper interventions. Hence, this study was conducted to understand major demographic, socioeconomic and landscape factors associated with leishmaniasis in Kurunegala district, which is one of the highest disease-endemic districts in Sri Lanka.

\section{Methods}

\section{Study area}

Kurunagala district $\left(7^{\circ} 45^{\prime} \mathrm{N}, 80^{\circ} 15^{\prime} \mathrm{E}\right)$ is located in the North-Western Province of Sri Lanka covering 4812.7 $\mathrm{km}^{2}$. Approximately 1,676,000 inhabitants live in the area within nearly 439,065 households [29]. About $32.6 \%$ of the population depends on agriculture-related employment while $36.6 \%$ are involved in other non-agricultural services followed by industry [30]. The prevalence of the disease in Sri Lanka is approximately $0.017 \%$. Kurunegala

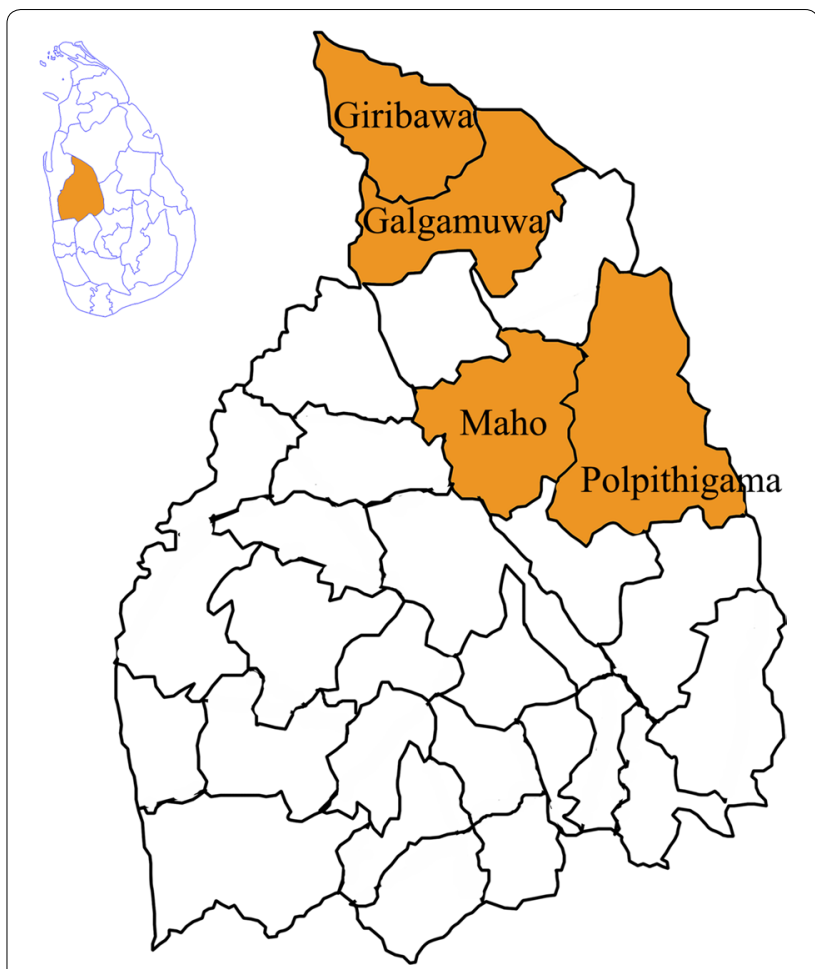

Fig. 1 The location of selected $\mathrm{MOH}$ areas within an extracted map of Kurunegala District, Sri Lanka

is one of the endemic districts with high prevalence of CL. According to patient records from 2009 to 2016, there was nearly an 18 -fold increase in the leishmaniasis incidence in this area. In 2018, a total of 533 cases were reported with an approximate prevalence of $0.37 \%$ [4].

\section{Selection of the study site}

The district of Kurunegala has 26 Medical Officer of Health $(\mathrm{MOH})$ areas. The prevalence of leishmaniasis in all $\mathrm{MOH}$ areas is not uniform. The disease prevalence is considerably higher in some $\mathrm{MOH}$ areas. Galamuwa, Giribawa Maho and Polpithigama MOH areas, which are on the list of high disease occurrences, were selected for the present study (Fig. 1).

For the case group, all patients in four selected areas during the period under study (2013-2016) were included. For the control group, a list of villages with previous records of patients was prepared and arranged alphabetically. This list was numbered consecutively, and villages were selected using a random number table. The selected villages were visited, and every third household was considered for the interview. If there were no records of a household member having CL, this household was included into the control group. This process was continued until the required sample size $(n=101$ matched with 101 cases, case:control =1:1) was achieved [31]. 


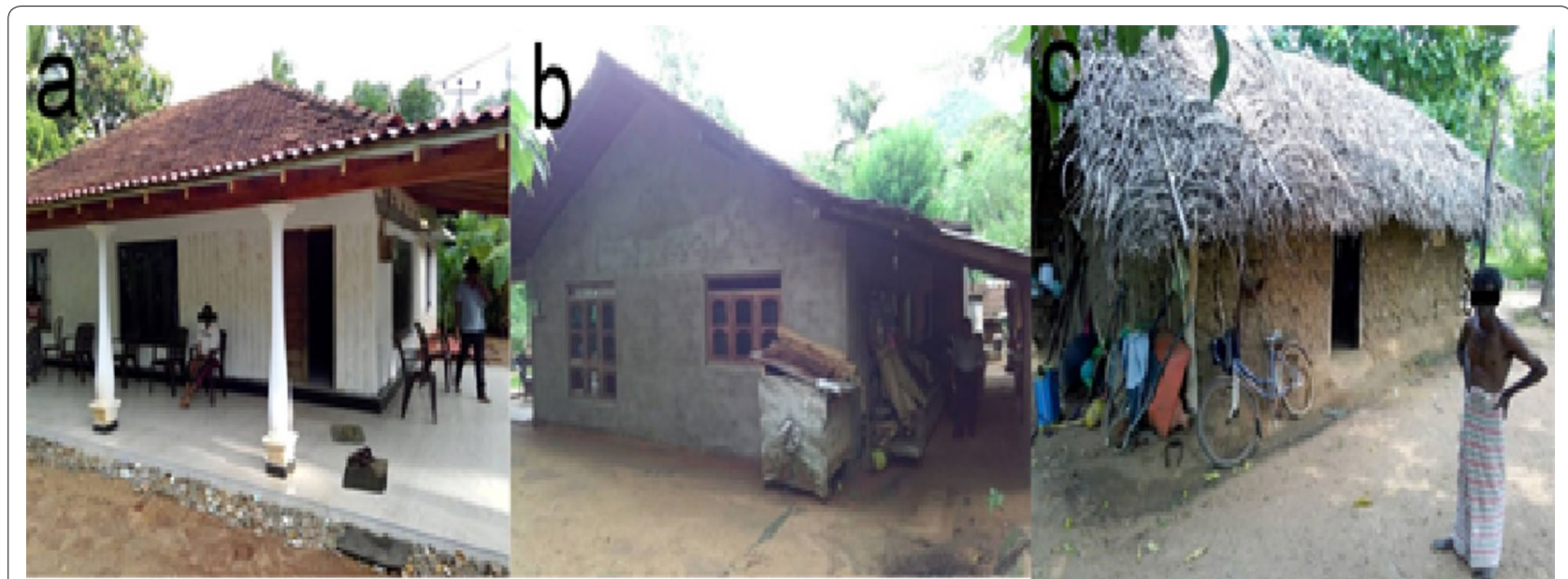

Fig. 2 Examples for house types. a Good (plastered cement walls with tiled or asbestos roofs). b Moderate (un-plastered brick walls with tiled or asbestos roofs). c Poor (clay walls, thatched, or aluminum sheet roof)

\section{Data collection}

Records of the leishmaniasis patients who reside in Galgamuwa, Giribawa, Maho and Polpithigama $\mathrm{MOH}$ areas that notified the relevant MOH office during 2013-2016 were obtained. Patient households were visited, and a pre-tested structured questionnaire was administered. If the patient was below 18 years-old, the parent or guardian was interviewed.

The basic demographic information such as age in years, level of education, marital status, gender and the family size of the respondent was recorded. Socioeconomic factors such as occupation, monthly income and house conditions were recorded. House conditions were categorized as "poor", "moderate" and "good" based on roof, wall and floor characteristics [32]. The houses having plastered cement walls with tiled or asbestos roofs were categorized as "good" (Fig. 2a) while un-plastered brick walls with tiled or asbestos roofs were considered as "moderate" (Fig. 2b). All other types of housing were grouped as "poor" (Fig. 2c).

The surroundings of the households were observed and landscape characteristics such as the presence of decaying garbage, termite hills, manna bushes, water streams, unclear areas (dark areas with trees or/and shrubs) and gardening areas within approximately a $100 \mathrm{~m}$ radius were recorded. These factors were selected based on the published literature and preliminary studies conducted in these areas [12, 33, 34]. In the awareness assessment, a series of questions were asked providing choices where applicable. However, guesses were not included as a response. In the recording of clinical features, clinical records available with the patient were referred to for the confirmation of responses given by patients.

\section{Data analysis}

Collected data were entered into EpiData version 4.4.3.1 (https://www.epidata.dk) data handling software package. These data were exported as CSV files and analyzed in IBM SPSS Statistics for Windows, Version 25.0 (IBM Corp., Armonk, NY, USA). The demographic, landscape and socioeconomic factors associated with CL in the case and control groups were compared by the Chi-square test for independence. Odds ratios were calculated with a 95\% confidence interval for each significantly different variable through univariate and multivariate regression analysis. For each categorical variable, the last category was selected as the reference category to calculate the odds ratio. The backward conditional logistic regression model was used for univariate analysis. The number of patients was selected as the dependent variable and the selected risk factor was considered as the independent variable. Nagelkerke $R^{2}$ value and the significance of the odds ratio were considered for the interpretation of univariate analysis results. For multivariate analysis, the multinomial logistic regression model was used. Age and gender were included as covariates to exclude the confounding effects. The number of patients was considered as the dependent variable and the selected risk factor was considered as the independent variable. The likelyhood ratio test and the goodness-of-fit-test were performed to assess the model.

\section{Results}

A total of 117 patients had been reported from all four $\mathrm{MOH}$ areas during 2013-2016. Among them, a total of 101 patients were included in the study. Other patients were excluded due to one or more of the reasons; inaccurate contact information provided by the patients, 


\section{Total of 117 cases were reported} to MOH offices

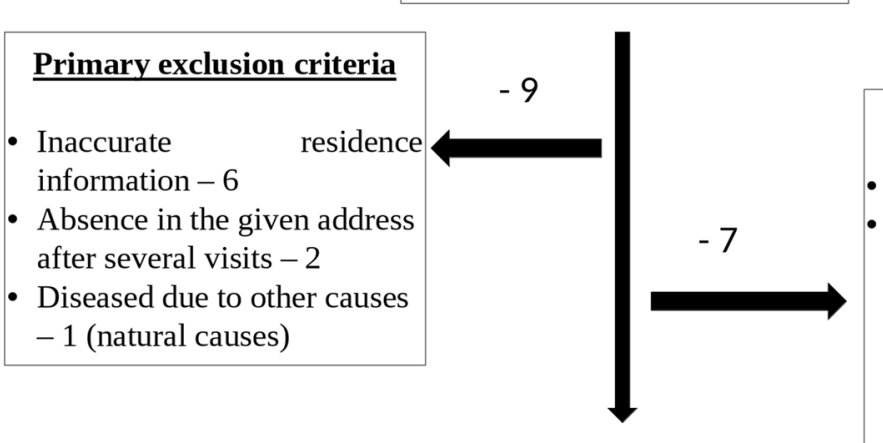

\section{Secondary exclusion criteria}

- Unwillingness to participate - 4

- An another disease inaccurately documented as leishmaniasis confirmed after examining medical records of the patients.

- Chronic kidney disease - 1

- Dengue - 3

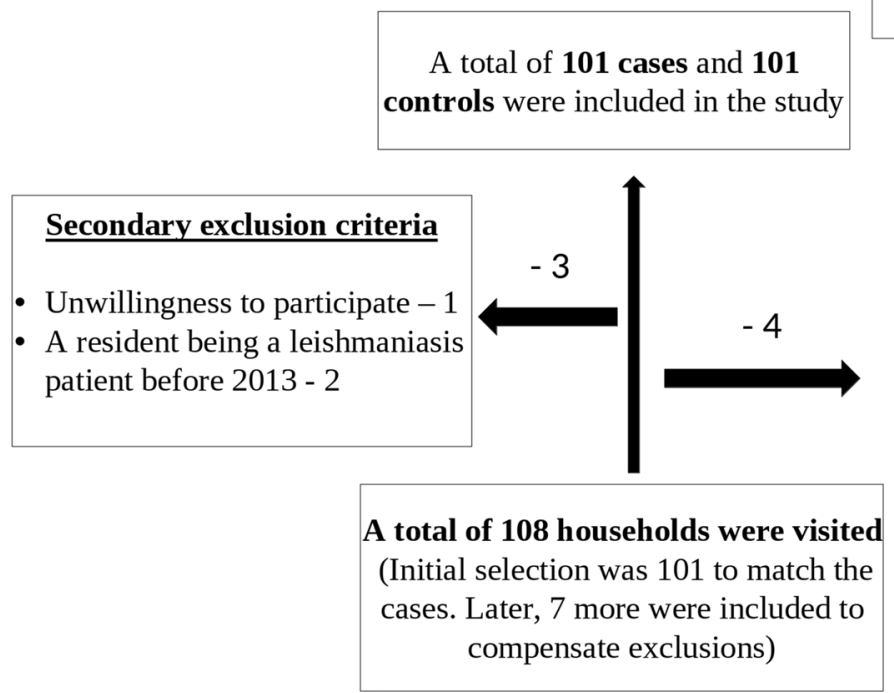

\section{Primary exclusion criteria}

- Absence of residents in the selected household - 4

Fig. 3 Inclusion and exclusion criteria of the cutaneous leishm
and Polpithigama MOH areas in Kurunegala District, Sri Lanka

absence at the provided address after 3 visits and change of residency to a different area, false-positive reports as confirmed after referring to medical records and unwillingness to participate (Fig. 3). The spatial distribution of cases and controls is illustrated in Fig. 3. The numbers of patients from each area are tabulated in the Additional file 1: Table S1.

\section{Clinical features}

All patients had the cutaneous form of the disease and none of the patients had visceral leishmaniasis or mucocutaneous leishmaniasis symptoms. The most common type of the lesion encountered was nodules $(n=64$, $63.36 \%)$ followed by ulcers $(n=31 ; 30.70 \%)$ and plaques $(n=6,5.94 \%)$. Having more than one lesion was rare and observed only in 12 patients. The majority of the lesions were less than $15 \mathrm{~mm}$ in diameter $(n=72,63.16 \%)$. The remainder were between $15-30 \mathrm{~mm}(n=34,29.82 \%)$ and rarely exceeded $30 \mathrm{~mm}(n=8,7.02 \%)$. The lesions were more prominent on the hands $(n=39,34.21 \%)$ and face $(n=37,32.45 \%)$ (Table 1$)$. Only one patient had a recurrent infection two months after completion of the treatment episode (Fig. 4).

\section{Demographic features}

Most of the patients were male $(n=56,55.44 \%)$ within the age group of $26-35$ years-old $(n=23,22.77 \%)$ which covers the working-age (Table 2). The majority of the patients were married $(n=84,83.17 \%)$ with an average of $1-3$ members in the family $(n=58,57.42 \%)$. Seventyfive patients $(67.32 \%)$ had received education at least up to the General Certificate of Education in Ordinary Level (GCE O/L). The Chi-square test indicated that the two groups (case and control) significantly differed with respect to age $(P<0.05)$, gender $(P<0.001)$ and marital status $(P<0.05)$. The size of the family and the level 
Table 1 Clinical features of patients confirmed for cutaneous leishmaniasis

\begin{tabular}{ll}
\hline Feature & No. of patients (\%) \\
\hline Lesion type & \\
Ulcers & $31(30.69)$ \\
Nodule & $64(63.37)$ \\
Plaque & $6(5.94)$ \\
Number of lesions & \\
1 & $89(88.12)$ \\
2 & $11(10.89)$ \\
3 & $1(0.99)$ \\
Lesion size (mm) & \\
$<15$ & $72(63.16)$ \\
$15-30$ & $34(29.82)$ \\
$>30$ & $8(7.92)$ \\
Lesion location & \\
Face & $37(32.46)$ \\
Earlobe & $9(7.89)$ \\
Torso & $14(12.28)$ \\
Legs & $15(13.15)$ \\
Hands & $39(34.22)$ \\
\hline
\end{tabular}

of education did not differ significantly among the two groups $(P>0.05)$ (Table 2$)$.

Univariate analysis comparing cases and controls showed that age does not increase or decrease the odds of having the disease at significant levels. However, children less than 15 years-old had higher odds of acquiring the disease (OR: 6.000, 95\% CI: 0.390-92.277) than the other two groups, though not statistically significant (Table 3). Interestingly, males had reduced odds of becoming infected than females (OR: 0.273 , 95\% CI: $0.144-0.520$ ). On the other hand, unmarried individuals were less likely to be leishmaniasis patients than married individuals (OR: 0.368, 95\% CI: 0.145-0.931). Although associations indicated by odds ratios remained the same for gender, after the adjustment on age in the multivariable conditional logistic regression model, it was no longer statistically significant.

\section{Socioeconomic characteristics}

Majority of the patients were farmers $(n=33,32.67 \%)$, followed by housewives $(n=29,28.71 \%)$. Most of the patients had a monthly income of Rs. 10,001-20,000 (56.76-113.52 USD) $(n=33,32.67 \%)$ (Table 2). Patients predominantly occupied houses with moderate conditions $(n=52,51.48 \%)$, followed by houses with good $(n=38,37.62 \%)$ and poor $(n=11,10.89 \%)$ conditions. Major occupations $\left(X^{2}=24.429, d f=10, P<0.05\right)$ and monthly income $\left(\chi^{2}=15.372, d f=3, P<0.05\right)$ were significantly different between case and control groups. However, the univariate conditional regression analysis suggested that being a housewife has elevated odds of becoming infected (OR: 3.623, 95\% CI: 0.915-14.286).

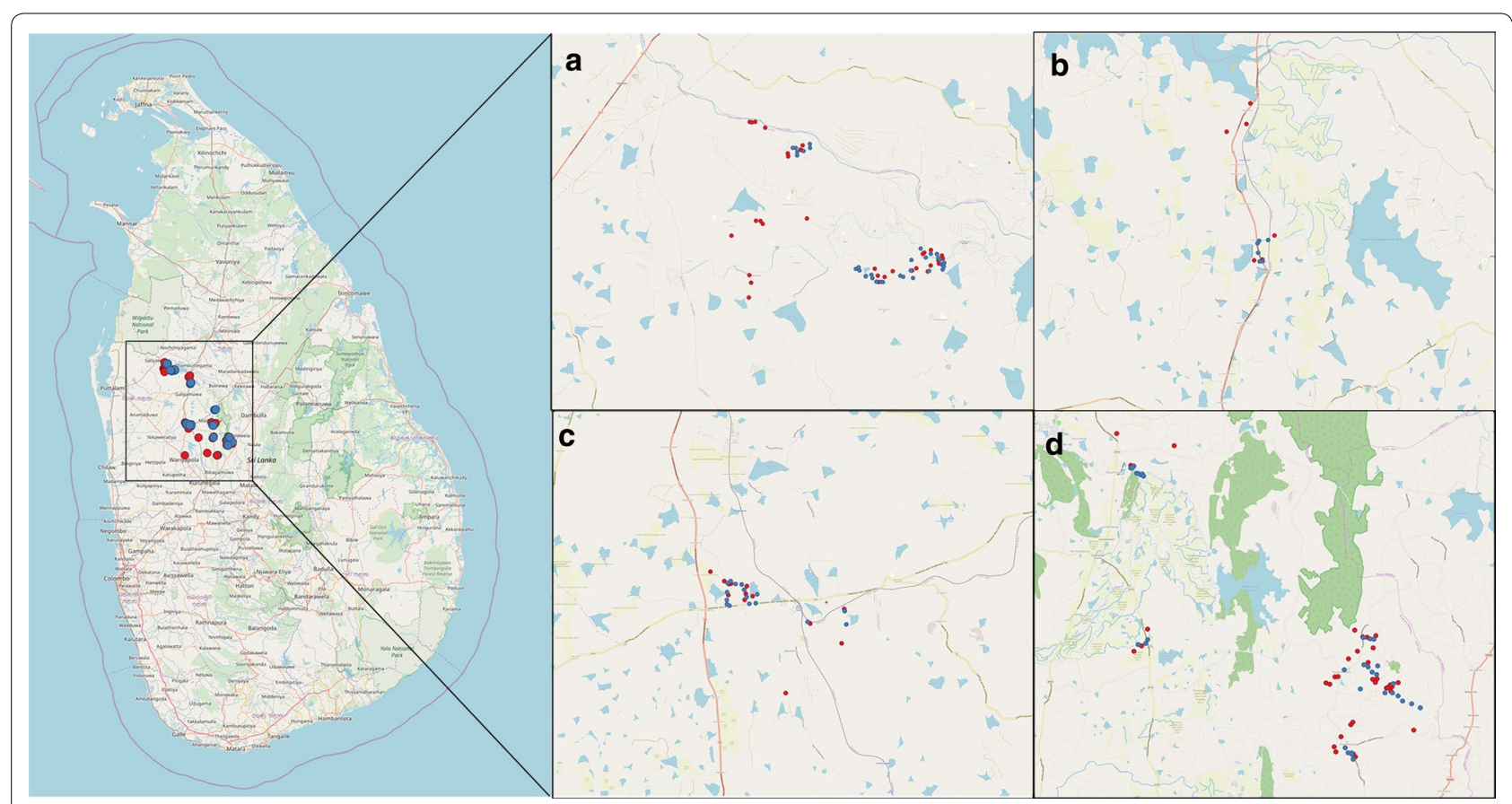

Fig. 4 Distribution of cutaneous leishmaniasis cases and controls in a map of Sri Lanka (1:4,000,000) and each MOH area (1:80,000): a Giribawa, b Galgamuwa, c Maho, d Polpithigama. Red dots indicate the locations of the patients while the blue dots indicate the location of controls 
Table 2 Results for the comparison of demographic, socioeconomic and landscape factors between the case and control groups by Chi-square test

\begin{tabular}{|c|c|c|c|c|}
\hline Factor & $\begin{array}{l}\text { Case } \\
n(\%)\end{array}$ & $\begin{array}{l}\text { Control } \\
n(\%)\end{array}$ & $x^{2}$ & $P$-value \\
\hline \multicolumn{5}{|l|}{ Age (years) } \\
\hline$<15$ & $9(8.91)$ & $1(0.99)$ & \multirow[t]{8}{*}{24.690} & \multirow[t]{8}{*}{0.001} \\
\hline $15-25$ & $8(7.92)$ & $5(4.95)$ & & \\
\hline $26-35$ & $23(22.77)$ & $51(50.49)$ & & \\
\hline $36-45$ & $22(21.78)$ & $21(20.79)$ & & \\
\hline $46-55$ & $18(17.82)$ & $13(12.87)$ & & \\
\hline $56-65$ & $10(9.90)$ & $7(6.93)$ & & \\
\hline $66-75$ & $8(7.92)$ & $1(0.99)$ & & \\
\hline $76-85$ & $3(2.97)$ & $2(1.98)$ & & \\
\hline \multicolumn{5}{|l|}{ Gender } \\
\hline Male & $56(55.45)$ & $82(81.19)$ & \multirow[t]{2}{*}{15.461} & \multirow[t]{2}{*}{$<0.00$} \\
\hline Female & $45(44.55)$ & $19(18.81)$ & & \\
\hline \multicolumn{5}{|l|}{ Marital status } \\
\hline Married & $84(83.17)$ & $94(93.07)$ & \multirow[t]{2}{*}{4.728} & \multirow[t]{2}{*}{0.030} \\
\hline Unmarried & $17(16.83)$ & $7(6.93)$ & & \\
\hline \multicolumn{5}{|l|}{ Family size } \\
\hline $1-3$ & $58(57.43)$ & $49(48.51)$ & \multirow[t]{3}{*}{1.668} & \multirow[t]{3}{*}{0.434} \\
\hline $4-6$ & $41(40.59)$ & $49(48.51)$ & & \\
\hline$>6$ & $2(1.98)$ & $3(2.98)$ & & \\
\hline \multicolumn{5}{|l|}{ Education } \\
\hline Grade 1-4 & $6(5.94)$ & $2(1.98)$ & \multirow[t]{6}{*}{4.259} & \multirow[t]{6}{*}{0.642} \\
\hline Grade 5 & $23(22.77)$ & $27(26.73)$ & & \\
\hline $\mathrm{O} / \mathrm{L}$ & $46(45.54)$ & $53(52.48)$ & & \\
\hline$A / L$ & $20(19.80)$ & $16(15.84)$ & & \\
\hline Higher education & $2(1.98$ & $1(0.99)$ & & \\
\hline None & $4(3.96)$ & $2(1.98)$ & & \\
\hline \multicolumn{5}{|l|}{ Major occupation } \\
\hline Armed forces & $3(2.98)$ & $6(5.94)$ & \multirow[t]{10}{*}{24.429} & \multirow[t]{10}{*}{0.005} \\
\hline Transport service worker (driver/conductor) & $6(5.94)$ & $4(3.96)$ & & \\
\hline Farmer & $33(32.67)$ & $29(28.71)$ & & \\
\hline Government server & $5(4.95)$ & $9(8.91)$ & & \\
\hline Labourer & $2(1.98)$ & $3(2.98)$ & & \\
\hline Mason & $6(5.94)$ & $16(15.84)$ & & \\
\hline Private sector employee & $3(2.98)$ & $7(6.93)$ & & \\
\hline Self employed & $8(7.92)$ & $13(12.87)$ & & \\
\hline Housewife & $29(28.71)$ & $8(7.92)$ & & \\
\hline Student & $6(5.94)$ & $6(5.94)$ & & \\
\hline \multicolumn{5}{|l|}{ Monthly income Rs. (USD) } \\
\hline$<10,000(<56.76)$ & $20(19.80)$ & $3(2.98)$ & \multirow[t]{4}{*}{15.372} & \multirow[t]{4}{*}{0.002} \\
\hline $10,001-20,000(56.76-113.52)$ & $33(32.67)$ & $41(40.59)$ & & \\
\hline $20,001-30,000(113.52-170.28)$ & $27(26.73)$ & $26(25.74)$ & & \\
\hline$>30,000(>170.28)$ & $21(20.79)$ & $31(30.69)$ & & \\
\hline \multicolumn{5}{|l|}{ House condition } \\
\hline Poor & $11(10.89)$ & $13(12.87)$ & \multirow[t]{3}{*}{2.745} & 0.253 \\
\hline Moderate & $52(51.49)$ & $61(60.40)$ & & \\
\hline Good & $38(37.62)$ & $27(26.73)$ & & \\
\hline
\end{tabular}


Table 2 (continued)

\begin{tabular}{|c|c|c|c|c|}
\hline Factor & $\begin{array}{l}\text { Case } \\
n(\%)\end{array}$ & $\begin{array}{l}\text { Control } \\
n(\%)\end{array}$ & $x^{2}$ & $P$-value \\
\hline \multicolumn{5}{|l|}{ Decaying garbage } \\
\hline Present & $57(56.44)$ & $25(24.75)$ & \multirow[t]{2}{*}{20.559} & \multirow[t]{2}{*}{$<0.001$} \\
\hline Absent & $44(43.56)$ & $76(75.25)$ & & \\
\hline \multicolumn{5}{|l|}{ Termite hills } \\
\hline Present & $45(44.55)$ & $34(33.66)$ & \multirow[t]{2}{*}{9.272} & \multirow[t]{2}{*}{$<0.05$} \\
\hline Absent & $56(55.45)$ & $67(66.34)$ & & \\
\hline \multicolumn{5}{|l|}{ Manna bushes } \\
\hline Present & $50(49.50)$ & $43(42.57)$ & \multirow[t]{2}{*}{0.741} & \multirow[t]{2}{*}{0.389} \\
\hline Absent & $51(50.50)$ & $58(57.43)$ & & \\
\hline \multicolumn{5}{|l|}{ Water streams } \\
\hline Present & $47(46.53)$ & $60(60.40)$ & \multirow[t]{2}{*}{3.358} & \multirow[t]{2}{*}{0.067} \\
\hline Absent & $54(53.47)$ & $41(39.60)$ & & \\
\hline \multicolumn{5}{|l|}{ Unclear areas } \\
\hline Present & $63(62.38)$ & $40(39.60)$ & \multirow[t]{2}{*}{10.070} & \multirow[t]{2}{*}{$<0.05$} \\
\hline Absent & $38(37.62)$ & $61(60.40)$ & & \\
\hline \multicolumn{5}{|l|}{ Areas with wet soil } \\
\hline Present & $52(51.49)$ & $30(29.70)$ & \multirow[t]{2}{*}{9.936} & \multirow[t]{2}{*}{$<0.05$} \\
\hline Absent & $49(48.51)$ & $71(70.30)$ & & \\
\hline \multicolumn{5}{|l|}{ Gardening areas } \\
\hline Present & $50(49.50)$ & $26(25.74)$ & \multirow[t]{2}{*}{11.806} & \multirow[t]{2}{*}{0.001} \\
\hline Absent & $51(50.50)$ & $75(74.26)$ & & \\
\hline \multicolumn{5}{|c|}{ Animals owned or frequently visiting the home harden and household } \\
\hline Dogs & $77(76.24)$ & $51(50.50)$ & 8.891 & 0.180 \\
\hline Cats & $36(35.64)$ & $17(16.83)$ & & \\
\hline Cattle & $8(7.92)$ & $8(7.92)$ & & \\
\hline Chicken & $2(1.98)$ & $4(3.96)$ & & \\
\hline Wild boar & $12(11.88)$ & $4(3.96)$ & & \\
\hline Monkeys & $2(1.98)$ & $0(0)$ & & \\
\hline Rodents & $13(12.87)$ & $3(2.98)$ & & \\
\hline Heard of the disease & & & & \\
\hline Yes & $25(24.75)$ & $66(65.35)$ & 33.617 & $<0.001$ \\
\hline No & $76(75.25)$ & $35(34.65)$ & & \\
\hline If yes, source & & & & \\
\hline TV/radio & $3(12.00)$ & $8(12.12)$ & 36.816 & $<0.001$ \\
\hline Printed media & $1(4.00)$ & $2(3.03)$ & & \\
\hline Friend & $19(76.00)$ & $39(59.09)$ & & \\
\hline Health worker & $2(8.00)$ & $17(25.75)$ & & \\
\hline Seen a patient? (bef & & & & \\
\hline Yes & $16(15.84)$ & $43(42.57)$ & 17.454 & $<0.001$ \\
\hline No & $85(84.16)$ & $58(57.43)$ & & \\
\hline If yes, source? & & & & \\
\hline Village & 15 (93.75) & $37(86.05)$ & 16.802 & $<0.001$ \\
\hline Outside village & $1(6.25)$ & $4(9.30)$ & & \\
\hline TV/printed media & $0(0.00)$ & $2(4.65)$ & & \\
\hline Causative organism & & & & \\
\hline Correct & $2(1.98)$ & $0(0)$ & 2.020 & 0.155 \\
\hline Wrong & $0(0)$ & $0(0)$ & & \\
\hline Unaware & $99(98.02)$ & $101(100)$ & & \\
\hline
\end{tabular}


Table 2 (continued)

\begin{tabular}{|c|c|c|c|c|}
\hline Factor & $\begin{array}{l}\text { Case } \\
n(\%)\end{array}$ & $\begin{array}{l}\text { Control } \\
n(\%)\end{array}$ & $x^{2}$ & $P$-value \\
\hline \multicolumn{5}{|c|}{ Mode of transmission } \\
\hline Correct & $20(19.80)$ & $1(0.99)$ & \multirow[t]{3}{*}{6.000} & \multirow[t]{3}{*}{0.199} \\
\hline Wrong & $3(2.98)$ & $8(7.92)$ & & \\
\hline Unaware & $78(77.22)$ & $92(91.09)$ & & \\
\hline \multicolumn{5}{|c|}{ Method of diagnosis } \\
\hline Correct & $1(0.99)$ & $0(0)$ & \multirow[t]{2}{*}{1.005} & \multirow[t]{2}{*}{0.316} \\
\hline Unaware & $100(99.01)$ & $101(100)$ & & \\
\hline \multicolumn{5}{|c|}{ Peak biting time } \\
\hline Correct & $5(4.95)$ & $0(0)$ & \multirow[t]{3}{*}{6.184} & \multirow[t]{3}{*}{0.045} \\
\hline Wrong & $1(0.99)$ & $0(0)$ & & \\
\hline Unaware & $95(94.06)$ & $101(100)$ & & \\
\hline \multicolumn{5}{|c|}{ Protective measures } \\
\hline Correct & $5(4.95)$ & $2(1.98)$ & \multirow[t]{3}{*}{2.451} & \multirow[t]{3}{*}{0.294} \\
\hline Wrong & $11(10.89)$ & $7(6.93)$ & & \\
\hline Unaware & $85(84.16)$ & $92(91.09)$ & & \\
\hline
\end{tabular}

Note: $P<0.05$ indicates a statistically significant result

According to the multivariate analysis, housewives were 3.9 times more likely to become infected (95\% CI: 0.60612.658). The individuals with a monthly income of $<$ Rs. 10,000 (56.76 USD) were 9.5 times more likely to become the infected than those who earn>Rs. 30,000 (170.28 USD) per month (Table 4).

\section{Landscape factors}

The presence of decaying garbage in the surroundings was observed amongst the majority of patient households ( $n=57,56.44 \%$ ). Moreover, many of the patient households ( $n=63,62.38 \%)$ had uncleared surroundings with pepper cultivations, banana plant aggregations and vegetation that provide shady and dark conditions. The number of patient households that had nearby wet soil areas was slightly higher $(n=52,51.49 \%)$. The main wet soil areas that were noted were the soil in open pits where collected water, turned soil in Chena cultivations, the soil surrounding cemented water tanks, the soil at the base of the cultivated plants which are regularly watered, paddy fields, and the ground surrounding animal huts. Among the landscape factors, presence or absence of decaying garbage, termite hills, unclear areas, areas with wet soil and gardening areas differed significantly between the case and control groups $(P<0.05)$. All these five factors were associated with increasing the risk of infection, according to the univariate analysis (Table 3). Multivariate logistic regression analysis indicated that the presence of decaying garbage increases the risk of infection by 4 times. The presence of termite hills, unclear areas, areas with wet soil and gardening areas increases the odds of having the disease by $2.4,2.7,2.4$ and 2.5 times, respectively.
Ownership of animals or animals which frequently visit the garden at home did not show a significant difference between the case and control groups $\left(\chi^{2}=8.891, d f=6\right.$, $P=0.180)$. However, the majority of the patients $(n=77$, $76.24 \%$ ) had pet or feral dogs in the surrounding area. Cats, cattle, chickens, wild boars, monkeys and rodents (rats, shrews and squirrels) were the other commonly visiting animals to home gardens in these areas.

\section{Awareness about the disease}

The majority of the patients $(n=76,75.25 \%)$ had not heard about a disease called leishmaniasis before infection, while most of the control group ( $n=66,65.35 \%)$ had heard about the disease. The majority had heard about the disease from a friend, and this was true for both case $(n=19)$ and control groups $(n=39)$. Awareness from health workers $(n=17,16.83 \%)$ was another source of information in the control group. Only 16 (15.84\%) individuals had seen someone else with the infection before they became infected (Table 2). Only two individuals who had the infection knew that the disease was caused by a protozoan parasite. No one in the control group was aware of the parasite.

A total of 20 patients mentioned that the mode of transmission is by sand flies. Only one person from the control group marked the correct answer that leishmaniasis is transmitted by the sand fly Phlebotomus argentipes. No one was aware of any diagnostic methods of leishmaniasis, except one patient who was aware of microscopy. Only five individuals in the case group correctly answered the question for peak biting time. Very few in the case $(n=5)$ and control groups $(n=2)$ were 
Table 3 Results of the univariate logistic regression analysis of the factors associated with the transmission of CL in Galgamuwa, Giribawa, Polpithigama and Maho MOH areas in the Kurunegala district, Sri Lanka

\begin{tabular}{|c|c|c|c|c|c|}
\hline Variable & Case & Control & OR & $95 \% \mathrm{Cl}$ & $R^{2}$ \\
\hline \multicolumn{6}{|l|}{ Age (years) } \\
\hline$<15$ & 9 & 1 & 6.000 & $0.390-92.277$ & \multirow[t]{8}{*}{0.165} \\
\hline $15-25$ & 8 & 5 & 1.067 & $0.129-8.793$ & \\
\hline $26-35$ & 23 & 51 & 0.204 & $0.047-1.923$ & \\
\hline $36-45$ & 22 & 21 & 0.709 & $0.106-4.607$ & \\
\hline $46-55$ & 18 & 13 & 0.935 & $0.134-6.335$ & \\
\hline $56-65$ & 10 & 7 & 0.962 & $0.125-7.275$ & \\
\hline $66-75$ & 8 & 1 & 0.232 & $0.343-82.827$ & \\
\hline $76-85$ & 3 & 2 & 1 & & \\
\hline \multicolumn{6}{|l|}{ Gender } \\
\hline Male & 56 & 82 & $0.273^{*}$ & \multirow[t]{2}{*}{$0.144-0.520$} & \multirow[t]{2}{*}{0.107} \\
\hline Female & 45 & 19 & 1 & & \\
\hline \multicolumn{6}{|l|}{ Marital status } \\
\hline Married & 84 & 94 & $0.368^{*}$ & $0.145-0.931$ & \multirow[t]{2}{*}{0.032} \\
\hline Unmarried & 17 & 7 & 1 & & \\
\hline \multicolumn{6}{|l|}{ Major occupation } \\
\hline Armed forces & 3 & 6 & 0.5 & $0.084-2.994$ & \multirow[t]{10}{*}{0.153} \\
\hline Transport service worker (driver/conductor) & 6 & 4 & 1.499 & $0.274-8.196$ & \\
\hline Farmer & 33 & 29 & 1.138 & $0.330-3.922$ & \\
\hline Government server & 5 & 9 & 0.556 & $0.115-2.681$ & \\
\hline Labourer & 2 & 3 & 0.667 & $0.080-5.525$ & \\
\hline Mason & 6 & 16 & 0.375 & $0.086-1.631$ & \\
\hline Private sector employee & 2 & 7 & 0.286 & $0.041-1.980$ & \\
\hline Self employed & 8 & 13 & 0.615 & $0.147-2.584$ & \\
\hline Housewife & 29 & 8 & $3.623^{*}$ & $0.915-14.286$ & \\
\hline Student & 6 & 6 & 1 & & \\
\hline \multicolumn{6}{|l|}{ Monthly household income in Rs. (USD) } \\
\hline$<10,000(<56.76)$ & 20 & 3 & $9.841^{*}$ & $2.593-37.357$ & \multirow[t]{4}{*}{0.107} \\
\hline $10,001-20,000(56.76-113.52)$ & 33 & 41 & 1.188 & $0.579-2.439$ & \\
\hline $20,001-30,000(113.52-170.28)$ & 27 & 26 & 1.533 & $0.708-3.319$ & \\
\hline$>30,000(>170.28)$ & 21 & 31 & 1 & & \\
\hline \multicolumn{6}{|l|}{ Decaying garbage } \\
\hline Present & 57 & 25 & $3.886^{*}$ & $2.134-7.079$ & 0.132 \\
\hline Absent & 44 & 76 & 1 & & \\
\hline \multicolumn{6}{|l|}{ Termite hills } \\
\hline Present & 45 & 34 & $2.408^{*}$ & $1.361-4.261$ & 0.061 \\
\hline Absent & 56 & 67 & 1 & & \\
\hline \multicolumn{6}{|l|}{ Unclear areas } \\
\hline Present & 63 & 40 & $2.487^{*}$ & $1.410-4.387$ & 0.066 \\
\hline Absent & 38 & 61 & 1 & & \\
\hline \multicolumn{6}{|l|}{ Areas with wet soil } \\
\hline Present & 52 & 30 & $2.512^{*}$ & $1.409-4.478$ & 0.065 \\
\hline Absent & 49 & 71 & & & \\
\hline \multicolumn{6}{|l|}{ Gardening areas } \\
\hline Present & 50 & 26 & $2.790^{*}$ & $1.542-5.050$ & 0.077 \\
\hline Absent & 51 & 75 & & & \\
\hline
\end{tabular}

*Statistically significant $(P<0.05)$

Abbreviations: $\mathrm{OR}$, odds ratio; $R^{2}$, Nagelkerke $R^{2}$ value 
Table 4 Results of the multinomial logistic regression of the factors associated with the transmission of CL in Galgamuwa, Giribawa, Polpithigama and Maho MOH areas in the Kurunegala district, Sri Lanka

\begin{tabular}{|c|c|c|c|c|c|}
\hline Variable & Case & Control & OR & $95 \% \mathrm{Cl}$ & LR test \\
\hline \multicolumn{6}{|l|}{ Age (years) } \\
\hline$<15$ & 9 & 1 & 0.245 & $0.015-3.996$ & \multirow[t]{8}{*}{$<0.001$} \\
\hline $15-25$ & 8 & 5 & 0.968 & $0.112-8.400$ & \\
\hline $26-35$ & 23 & 51 & 3.957 & $0.588-26.640$ & \\
\hline $36-45$ & 22 & 21 & 1.541 & $0.222-10.701$ & \\
\hline $46-55$ & 18 & 13 & 1.165 & $0.162-8.386$ & \\
\hline $56-65$ & 10 & 7 & 1.472 & $0.180-12.030$ & \\
\hline $66-75$ & 8 & 1 & 0.261 & $0.016-4.306$ & \\
\hline $76-85$ & 3 & 2 & 1 & & \\
\hline \multicolumn{6}{|l|}{ Gender } \\
\hline Male & 56 & 82 & 0.273 & $0.144-0.521$ & \multirow[t]{2}{*}{$<0.001$} \\
\hline Female & 45 & 19 & 1 & & \\
\hline \multicolumn{6}{|l|}{ Marital status } \\
\hline Married & 84 & 94 & 0.350 & $0.122-1.001$ & \multirow[t]{2}{*}{$<0.001$} \\
\hline Unmarried & 17 & 7 & 1 & & \\
\hline \multicolumn{6}{|l|}{ Major occupation } \\
\hline Armed forces & 3 & 6 & 0.756 & $0.117-4.878$ & \multirow[t]{10}{*}{0.001} \\
\hline Transport service worker (driver/conductor) & 6 & 4 & 2.278 & $0.380-13.700$ & \\
\hline Farmer & 33 & 29 & 1.441 & $0.368-5.650$ & \\
\hline Government server & 5 & 9 & 0.585 & $0.111-3.067$ & \\
\hline Labourer & 2 & 3 & 1.016 & $0.112-9.259$ & \\
\hline Mason & 6 & 16 & 0.516 & $0.107-2.481$ & \\
\hline Private sector employee & 2 & 7 & 0.297 & $0.040-2.198$ & \\
\hline Self employed & 8 & 13 & 0.818 & $0.170-3.940$ & \\
\hline Housewife & 29 & 8 & $3.937^{*}$ & $0.606-12.658$ & \\
\hline Student & 6 & 6 & 1 & & \\
\hline \multicolumn{6}{|l|}{ Monthly household income in Rs. (USD) } \\
\hline$<10,000(<56.76)$ & 20 & 3 & $9.524^{*}$ & $2.439-37.037$ & \multirow[t]{4}{*}{$<0.001$} \\
\hline $10,001-20,000(56.76-113.52)$ & 33 & 41 & 1.048 & $0.493-2.232$ & \\
\hline $20,001-30,000(113.52-170.28)$ & 27 & 26 & 1.260 & $0.559-2.841$ & \\
\hline$>30,000(>170.28)$ & 21 & 31 & 1 & & \\
\hline \multicolumn{6}{|l|}{ Decaying garbage } \\
\hline Present & 57 & 25 & \multirow[t]{2}{*}{$4.131^{*}$} & $2.188-7.153$ & \multirow[t]{2}{*}{$<0.001$} \\
\hline Absent & 44 & 76 & & & \\
\hline \multicolumn{6}{|l|}{ Termite hills } \\
\hline Present & 45 & 34 & \multirow[t]{2}{*}{$2.427^{*}$} & $1.337-4.407$ & \multirow[t]{2}{*}{0.003} \\
\hline Absent & 56 & 67 & & & \\
\hline \multicolumn{6}{|l|}{ Unclear areas } \\
\hline Present & 63 & 40 & $2.742^{*}$ & $1.502-5.005$ & \multirow[t]{2}{*}{0.001} \\
\hline Absent & 38 & 61 & 1 & & \\
\hline \multicolumn{6}{|l|}{ Areas with wet soil } \\
\hline Present & 52 & 30 & $2.492^{*}$ & $1.360-4.564$ & \multirow[t]{2}{*}{0.003} \\
\hline Absent & 49 & 71 & 1 & & \\
\hline \multicolumn{6}{|l|}{ Gardening areas } \\
\hline Present & 50 & 26 & $2.577^{*}$ & $1.389-4.779$ & 0.002 \\
\hline Absent & 51 & 75 & 1 & & \\
\hline
\end{tabular}

*Statistically significant $(P<0.05)$

Abbreviations: $\mathrm{OR}$, odds ratio; $\mathrm{LR}$, likelihood ratio 
aware of the correct protective measures to prevent vector biting, other individuals failed to reply. No significant difference between case and control groups was observed regarding knowledge about the disease, except for the peak biting time, which was higher for individuals who had had the disease previously. However, hearing about the disease and seeing a patient with the disease was significantly different between the two groups, where only very few from the case group had heard about the disease $(n=25)$ or had seen a patient before they acquired the infection $(n=16)$.

\section{Discussion}

The South Asian region has suffered from the devastating epidemics of leishmaniasis since the early 1980s [35]. Nonetheless, substantial progress has been made in controlling leishmaniasis in this region through successful diagnosis via active detection, complete case management, effective vector control measures, community mobilization and operational use of research generated knowledge [36, 37]. In Sri Lanka, leishmaniasis has received little attention from the authorities despite regular increases in the number of cases every year since 2011 [38]. One of the main limitations in the control of this disease in Sri Lanka is the lack of research at the regional level that would highlight the important risk factors that need to be addressed through control programmes. In this regard, the present study revealed valuable information.

The demographic factors associated with CL differ regionally, in some areas the elderly population is mostly affected [39], or in other areas the younger population [40]. The younger population was the most affected group according to the univariate analysis. However, this did not significantly predict the disease incidence. Although gender and marital status showed statistically significant associations according to the univariate analysis, it did not significantly predict the disease incidence as indicated by the adjusted odds ratios. Hence, the observed significant relationships in the univariate analysis are likely to be a result of confounding effects [41]. Therefore, demographic factors may not significantly affect the odds of having the disease.

In general, outdoor occupations increase the risk of leishmaniasis incidence due to a greater risk of being bitten by sand flies $[12,42,43]$. Furthermore, the results of previous studies in Sri Lanka have indicated that $P$. argentipes is more likely to transmit the parasite to humans as outdoor bites [6]. As observed in the present study and previous studies [27, 40,44], housewives are at risk of receiving infection. This may be due to their outside routine activities such as picking firewood, cleaning home gardens, washing clothes and bathing at outdoor water sources. Furthermore, women of farming families often denominate themselves as housewives, but they tend to assist their husbands in farming activities, which make them equally prone to vector sand fly bites. Therefore, this study indicates the importance of more extensive studies to assess the effects of day to day activities on the acquisition of this infection.

Leishmaniasis is considered a neglected tropical disease, where the poorest of the population is affected [45]. This has been demonstrated in the present study, as the odds of infection with leishmaniasis increased with poverty. Those who had a monthly income of $<$ Rs. 10,000 (56.76 USD) were potentially at risk of infection 9.5 times higher compared to those who had a monthly income of $>$ Rs. 30, 000 (170.27 USD). Generally, neglected tropical diseases affect poor populations due to poor sanitation, poor housing conditions, and lack of access to essential nutrition [46]. In this case, housing condition was not a significant factor in the both case and control groups. Therefore, in these study areas, house condition can be excluded as a major risk factor for the disease. However, with this knowledge, further studies would provide a better insight into other associated socioeconomical risk factors.

In any vector-borne disease, the presence of potential breeding/resting places of the vectors and reservoirs are associated with an increase in disease incidence [28, 46, 47]. In the present study, presence of potential breeding places for sand flies (decaying garbage, termite hills and areas with wet soil) and potential adult resting places (gardening areas and unclear areas) were associated with increased odds of leishmaniasis incidence. This is consistent with previous studies which indicated a higher risk of acquiring leishmaniasis with the presence of suitable resting and breeding sites in close proximity [11, 47]. Although reservoir hosts of the parasite in Sri Lanka is unclear, dogs are suspected to be a reservoir host [9, 10]. Nevertheless, the presence or absence of dogs, other pets (cats), livestock (cattle and chicken), stray animals (wild boar, rodents and monkeys) commonly visiting home gardens did not differ significantly between the two groups $(P>0.05)$. The same scenario has been observed from another study conducted in a different region of Sri Lanka [11]. The absence of increased exposure to potential reservoirs by patients is not adequate in making a strong conclusion about the role of reservoir hosts of the disease. The parasite $L$. donovani is known to have an anthroponotic transmission cycle, according to studies from other countries [48-50]. A study suggested the possibility of cattle being a reservoir host for L. donovani in Bangladesh [51]. However, more evidence indicates that the transmission may not involve an animal reservoir [48-50]. In align with these findings, studies from Sri 
Lanka show no infections in rodents and low infection rates in dogs $[9,10]$. Therefore, it is more likely that the parasite has an anthroponotic transmission cycle in this area of Sri Lanka. However, further studies on the interactions between the reservoir host, vector, parasite and human hosts are essential for a better understanding of the involvement of a reservoir in disease transmission.

Awareness about the disease in both control and case groups was very poor. No one without a history of leishmaniasis infection knew about the causative organism, while only two individuals knew that the disease is caused by a parasite. Some of the infected individuals mentioned the name of the vector correctly. But, none of them were aware what kind of insect it was. When they were shown a live specimen, they identified it by another name other than the Sinhalese term coined by the scientific community. Villagers used the same Sinhalese term for both biting midges and sand flies ("Ho haputta"). Researchers used the exact Sinhalese translation of the term sand fly ("Weli massa") to introduce sand flies. This often confused the villagers, as it is not a name which is commonly known among the community. The Sinhala term for sand flea ("Weli makka") is very similar to the Sinhala term used for sand flies. Therefore, inhabitants in these disease-endemic areas often misinterpret sand flea as the vector of leishmaniasis. Hence, the majority of the incorrect responses during the study was sand flea as the disease vector. In two cases, inhabitants believed that the disease can be transmitted by the fruit fly and the lesion is due to the eggs laid by the fruit fly on the skin. This assumption, which they believed to be true, was based on their experience in vegetable and fruit cultivations. Knowledge of protective measures was also very poor among the study population. Only a few $(n=7)$ respondents were aware of the correct protective measures such as wearing protective clothing, the use of insect repellents and minimizing outdoor activities during dusk/dawn. One of the most common responses was the use of mosquito nets, which was considered as a wrong response, as sand flies can crawl through the mesh of mosquito nets [52, 53]. Another incorrect response was staying away from sand to avoid sand fly biting, and thereby to prevent from the infection.

In statistical analyses, the two groups were initially compared using the Chi-square test. Statistically significant values were assessed by univariate logistic regression. However, the results may be affected by confounding factors. Therefore, the odds ratios adjusted for age and gender were calculated by multivariate logistic regression. However, certain factors showed significant differences between the two groups according to the Chi-square test but did not show a relationship once adjusted for age and gender. Therefore, initially existing relationships could be attributed to the confounding effects.

During the survey, the respondents were asked to recall the time before acquiring infection. This may have resulted in inaccuracies of the information provided. Furthermore, patients may not always provide correct answers about their education level and income due to social stigma. Another caveat of this study is that we recorded only the chief occupation of the patients. Most of the inhabitants were involved in agriculture-related activities in addition to their main occupation. Therefore, monitoring of their day to day activities, other than the main occupation is required. Furthermore, depending on the number of inhabitants in the study area, the ideal sample size for the study would be above 383 (at the $95 \%$ confidence level) [54]. Therefore, having only 101 patients for the study period is another limitation of this study. However, despite these unavoidable limitations, this study addresses some important aspects of the perception of the disease among at-risk communities. Therefore, the findings would be useful in implementing control programmes through a systematized action plan under the Ministry of Health, which has been identified as of paramount importance at present.

\section{Conclusions}

This study demonstrates that the occupation, monthly income, and the presence of potential breeding and resting places in the environment are the main risk factors for the disease transmission in these areas. Among them, housewives, low-income individuals, inhabitants of households with decaying garbage, termite hills, unclear areas, areas with wet soil and gardening areas in the surrounding are at higher risk. Further, poor awareness about the disease among the general public is also a major negative point. Hence, the control efforts must be focused on raising awareness about protective measures, other important aspects and improvement of environmental/landscape conditions while implementing major control activities such as vector control, reservoir host control and effective case management.

\section{Supplementary information}

Supplementary information accompanies this paper at https://doi. org/10.1186/s13071-020-04122-1.

Additional file 1: Table S1. The number of patients responded to the survey as the case group along with the percentage respondents of the total recorded patients identified from each Medical Officer of Health $(\mathrm{MOH})$ area. 


\section{Abbreviations}

CL: cutaneous leishmaniasis; MOH: Medical Officer of Health; USD: United States dollar.

\section{Acknowledgments}

The National Research Council, Sri Lanka (grant No. NRC 16-142) is acknowledged for financial support. The staff of the Medical Officer of Health in Galgamuwa, Giribawa, Maho, and Polpithigama is acknowledged for their support during the fieldwork.

\section{Authors' contributions}

NG, NK and WR designed the study. TW performed data collection, data analysis and drafting of the manuscript. NG made critical revisions, contributed to data collection and overall supervision of research work. All authors read and approved the final manuscript.

\section{Funding}

This study was funded by the National Research Council, Sri Lanka (grant number NRC 16-142).

\section{Availability of data and materials}

Data supporting the conclusions of this article are included within the article and its additional file. A coded version of the dataset generated during the study will be available from the corresponding author upon reasonable request.

\section{Ethics approval and consent to participate}

The study was approved by the Ethics Review Committee, Faculty of Medicine, University of Kelaniya (Reference No. P/204/12/2016). Written consent was obtained from all the participants before the interview.

\section{Consent for publication}

Not applicable.

\section{Competing interests}

The authors declare that they have no competing interests.

\section{Author details}

1 Department of Parasitology, Faculty of Medicine, University of Kelaniya, Ragama, Sri Lanka. ${ }^{2}$ Biotechnology Unit, Industrial Technology Institute, Colombo 07, Sri Lanka.

Received: 1 September 2019 Accepted: 6 May 2020

Published online: 12 May 2020

\section{References}

1. WHO. Leishmaniasis. 2019. Geneva: World Health Organisation; 2019. https://www.who.int/news-room/fact-sheets/detail/leishmaniasis. Accessed 26 Apr 2019.

2. Galgamuwa LS, Sumanasena B, Yatawara L, Wickramasinghe S, Iddawela D. Clinico-epidemiological patterns of cutaneous leishmaniasis patients attending the Anuradhapura teaching hospital, Sri Lanka. Korean J Parasitol. 2017:55:1-7.

3. Siriwardana HVYD, Thalagala N, Karunaweera ND. Clinical and epidemiological studies on the cutaneous leishmaniasis caused by Leishmania (Leishmania) donovani in Sri Lanka. Ann Trop Med Parasitol. 2010:104:213-23.

4. Weekly epidemiological report. Epidemiology Unit, Sri Lanka. 2019 http:// www.epid.gov.Ik/web/index.php?option=com_content\&view=artic le\&id=148\&ltemid=449\&lang=en. Accessed 26 Jul 2019.

5. Karunaweera ND, Pratlong F, Siriwardane HV, Ihalamulla RL. Sri Lankan cutaneous leishmaniasis is caused by Leishmania donovani zymodeme MON-37. Trans R Soc Trop Med Hyg. 2003;97:380-1.

6. Wijerathna ACT, Gunathilaka PADHN, Fujii Y, Gunawardana NK, Gunasekara DC. Molecular detection of Leishmania donovani parasites in wild caught Phlebotomus argentipes using a PCR assay and species abundance of sand flies (Diptera: Psychodidae) in Kuruneagala District; an endemic focus of cutaneous leishmaniasis in Sri Lanka. Abstract presented at: 5th International Conference on Multidisciplinary Approaches;
Aug 31st-2nd Sept 2018; Faculty of Graduate Studies, University of Sri Jayewardenepura, Sri Lanka; 2018.

7. Wijerathna T, Gunathilaka N, Gunawardena K, Rodrigo W. Breeding sites of phlebotomine sand flies in cutaneous leishmaniasis endemic areas of the Kurunegala District in Sri Lanka. Abstract presented at: 3rd International Research Symposium on Pure and Applied Sciences; 26th Oct 2018; Faculty of Science, University of Kelaniya, Sri Lanka; 2018.

8. Wijerathna T, Gunathilaka N, Gunawardena K, Rodrigo W. Diurnal resting sites and potential breeding habitats of leishmaniasis vector sand flies in selected cutaneous leishmaniasis endemic regions in Kurunegala District, Sri Lanka. Abstract presented at: 2nd International Conference on Tropical Medicine; 8-9th Dec 2017; Centre For Research In Tropical Medicine, University of Peradeniya, Sri Lanka; 2017.

9. Nawaratna SSK, Weilgama DJ, Rajapaksha K. Cutaneous leishmaniasis in Sri Lanka: a study of possible animal reservoirs. Int J Infect Dis. 2009:13:513-7.

10. Rosypal AC, Tripp S, Kinlaw C, Hailemariam S, Tidwell RR, Lindsay DS, et al. Surveillance for antibodies to Leishmania spp. in dogs from Sri Lanka. J Parasitol. 2010;96:230-1.

11. Cerbino Neto J, Werneck GL, Costa CHN. Factors associated with the incidence of urban visceral leishmaniasis: an ecological study in Teresina, Piauí State. Brazil. Cad Saude Publica. 2009;25:1543-51.

12. Ranasinghe $S$, Wickremasinghe $R$, Munasinghe A, Hulangamuwa $S$, Sivanantharajah S, Seneviratne K, et al. Cross-sectional study to assess risk factors for leishmaniasis in an endemic region in Sri Lanka. Am J Trop Med Hyg. 2013;89:742-9.

13. Okindo EG, Kasili S, Kutima IHL, Mutai MJ. Socioeconomic impacts of leishmaniases on households of Marigat Sub County, Baringo County of Kenya. J Trop Dis. 2016:4:5.

14. Nazari M, Taravatmanesh G, Kaveh MH, Soltani A, Ghaem H. The effect of educational intervention on preventive behaviors towards cutaneous leishmaniasis at Kharameh City in 2014. Shiraz E Med J. 2016:17:4-10.

15. Ranjan A, Sur D, Singh VP, Siddique NA, Manna B, Lal CS, et al. Risk factors for Indian kala-azar. Am J Trop Med Hyg. 2005;73:74-8.

16. Bern C, Hightower AW, Chowdhury R, Ali M, Amann J, Wagatsuma $Y$, et al. Risk factors for kala-azar in Bangladesh. Emerg Infect Dis. 2005:11:655-62.

17. Barnett PG, Singh SP, Bern C, Hightower AW, Sundar S. Virgin soil: the spread of visceral leishmaniasis into Uttar Pradesh, India. Am J Trop Med Hyg. 2005;73:720-5.

18. Costa CH, Werneck GL, Rodrigues L Jr, Santos MV, Araújo IB, Moura LS, et al. Household structure and urban services: neglected targets in the control of visceral leishmaniasis. Ann Trop Med Parasitol. 2005;99:229-36.

19. Tesh RB. Control of zoonotic visceral leishmaniasis: is it time to change strategies? Am J Trop Med Hyg. 1995;52:287-92.

20. Armijos R, Weigel M, Izurieta R, Racines J, Zurita C, Herrera W, Vega M. The epidemiology of cutaneous leishmaniasis in subtropical Ecuador. Trop Med Int Health. 1997:2:140-52.

21. Assoian RK, Fleurdelys BE, Stevenson HC, Miller PJ, Madtes DK, Raines EW, et al. Expression and secretion of type beta transforming growth factor by activated human macrophages. Proc Natl Acad Sci USA. 1987;84:6020-4.

22. Bebo BF, Schuster JC, Vandenbark AA, Offner $\mathrm{H}$. Androgens alter the cytokine profile and reduce encephalitogenicity of myelin-reactive T cells. J Immunol. 1999;162:35-40.

23. Soares L, Abad-Franch F, Ferraz G. Epidemiology of cutaneous leishmaniasis in central Amazonia: a comparison of sex-biased incidence among rural settlers and field biologists. Trop Med Int Health. 2014;19:988-95.

24. AISamarai AM, AlObaidi HS. Cutaneous leishmaniasis in Iraq. J Infect Dev Countr. 2009:3:123-9.

25. Lima ID, Lima AL, de Oliveira Mendes-Aguiar C, Coutinho JF, Wilson $\mathrm{ME}$, Pearson RD, et al. Changing demographics of visceral leishmaniasis in northeast Brazil: lessons for the future. PLoS Negl Trop Dis. 2018;12:e0006164.

26. Iddawela D, Vithana SM, Atapattu D, Wijekoon L. Clinical and epidemiological characteristics of cutaneous leishmaniasis in Sri Lanka. BMC Infect Dis. 2018;18:108.

27. Davies CR, Gavgani AM. Age, acquired immunity and the risk of visceral leishmaniasis: a prospective study in Iran. Parasitology. 1999;119:247-57.

28. Desjeux P. The increase in risk factors for leishmaniasis worldwide. Trans $R$ Soc Trop Med Hyg. 2001;95:239-43. 
29. Department of Census and Statistics Sri Lanka. Census of population and housing 2012: final report. Department of Census and Statistics, Ministry of Finance and Planning, Sri Lanka. 2013. http://www.statistics.gov.lk/ PopHouSat/CPH2011/Pages/Activities/Reports/CPH_2012_5Per_Rpt.pdf. Accessed 27 Jul 2019.

30. Department of Census and Statistics, Sri Lanka. Household income and expenditure survey 2012/13: final report. Department of Census and Statistics, Ministry of Finance and Planning, Sri Lanka. 2013. http://www. statistics.gov.Ik/HIES/HIES2012 13FinalReport.pdf. Accessed 27 Jul 2019.

31. Oliveira CD, Diez-Roux A, César CC, Proietti FA. A case-control study of microenvironmental risk factors for urban visceral leishmaniasis in a large city in Brazil, 1999-2000. Rev Panam Salud Publica. 2006;20:369-76.

32. Gunathilaka N, Abeyewickreme W, Hapugoda M, Wickremasinghe R. Determination of demographic, epidemiological, and socioeconomic determinants and their potential impact on malaria transmission in Mannar and Trincomalee districts of Sri Lanka. Malar J. 2016;15:330.

33. Ryan JR, Mbui J, Rashid JR, Wasunna MK, Kirigi G, Magiri C, et al. Spatial clustering and epidemiological aspects of visceral leishmaniasis in two endemic villages, Baringo District, Kenya. Am J Trop Med Hyg. 2006;74:308-17.

34. Weerakoon HS, Ranawaka RR, Warnasekara J, Bandara P. Analysis of gaps in the correct diagnosis of leishmaniasis at primary care level; sociodemographic and environmental risk factors of leishmaniasis transmission in Anuradhapura. Anuradhapura Med. 2018;12:10-6.

35. Peters W, Prasad LSN. Kala-azar in India - its importance as an issue in public health. In: Proceedings of the Indo-UK Workshop on Leishmaniasis: 6-10 December 1983. New Delhi: India; 1983.

36. Singh OP, Hasker E, Boelaert M, Sundar S. Elimination of visceral leishmaniasis on the Indian subcontinent. Lancet Infect Dis. 2016;16:e304-9.

37. Fitzpatrick A, Al-Kobaisi NS, Maya JB, Chung YR, Duhan S, Elbegdorj E, et al. Sustaining visceral leishmaniasis elimination in Bangladesh - could a policy brief help? PLoS Negl Trop Dis. 2017;11:e0006081.

38. Wijerathna T, Gunathilaka N, Gunawardana K, Rodrigo W. Potential challenges of controlling leishmaniasis in Sri Lanka at a disease outbreak. Biomed Res Int. 2017;2017:6931497.

39. Reithinger R, Mohsen M, Aadil K, Sidiqi M, Erasmus P, Coleman PG. Anthroponotic cutaneous leishmaniasis, Kabul. Afghanistan. Emerg Infect Dis. 2003;9:727-9.

40. Gurel MS, Ulukanligil M, Ozbilge H. Cutaneous leishmaniasis in Sanliurfa: epidemiologic and clinical features of the last four years (1997-2000). Int J Dermatol. 2002;41:32-7.

41. Pourhoseingholi MA, Baghestani AR, Vahedi M. How to control confounding effects by statistical analysis. Gastroenterol Hepatol Bed Bench. 2012;5:79-83.

42. Abdellatif MZM, El-Mabrouk K, Ewis AA. An epidemiological study of cutaneous leishmaniasis in Al-jabal Al-gharbi, Libya. Korean J Parasitol. 2013;51:75-84.
43. Eid D, Guzman-Rivero M, Rojas E, Goicolea I, Hurtig AK, Illanes D, et al. Risk factors for cutaneous leishmaniasis in the rainforest of Bolivia: a crosssectional study. Trop Med Health. 2018;46:9.

44. Alam E, Abbas O, Moukarbel R, Khalifeh I. Cutaneous leishmaniasis: an overlooked etiology of midfacial destructive lesions. PLoS Negl Trop Dis. 2016;10:e0004426.

45. WHO. Neglected tropical diseases. Geneva: World Health Organisation; 2019. https://www.who.int/neglected_diseases/en/. Accessed 27 Jul 2019.

46. Stresman GH. Beyond temperature and precipitation: ecological risk factors that modify malaria transmission. Acta Trop. 2010;116:167-72.

47. Ferro C, Cadena H, Ocampo CB, Alexander N, Munstermann LE, Holford TR, et al. Environmental risk factors for the incidence of American cutaneous leishmaniasis in a sub-andean zone of Colombia (Chaparral, Tolima). Am J Trop Med Hyg. 2010;82:243-50.

48. WHO. Control of the leishmaniasis: report of a meeting of the WHO expert committee on the control of leishmaniases. Geneva: World Health Organization; 2010. https://apps.who.int/iris/bitstream/handle/10665 /44412/WHO_TRS_949_eng.pdf;jsessionid=1080644455DCDF800E6A 96814A4E57D1?sequence=1. Accessed 18 Dec 2019.

49. Antoniou M, Haralambous C, Mazeris A, Pratlong F, Dedet JP, Soteriadou K. Leishmania donovani leishmaniasis in Cyprus. Lancet Infect Dis. 2008;8:6-7.

50. Singh R, Kumar D, Ramesh V, Negi NS, Singh S, Salotra P. Visceral leishmaniasis, or kala azar (KA): high incidence of refractoriness to antimony is contributed by anthroponotic transmission via post-KA dermal leishmaniasis. J Infect Dis. 2006;194:302-6.

51. Alam MS, Ghosh D, Khan MGM, et al. Survey of domestic cattle for antiLeishmania antibodies and Leishmania DNA in a visceral leishmaniasis endemic area of Bangladesh. BMC Vet Res. 2011;7:27.

52. Reyburn $\mathrm{H}$, Ashford R, Mohsen M, Hewitt S, Rowland M. A randomized controlled trial of insecticide-treated bednets and chaddars or top sheets, and residual spraying of interior rooms for the prevention of cutaneous leishmaniasis in Kabul, Afghanistan. Trans R Soc Trop Med Hyg. 2000:94:361-6.

53. Unsworth K, Gordon RM. The maintenance of a colony of Phlebotomus papatasii in Great Britain. Ann Trop Med Parasitol. 1946;40:219-27.

54. Johnston KM, Lakzadeh P, Donato BMK, Szabo SM. Methods of sample size calculation in descriptive retrospective burden of illness studies. BMC Med Res Methodol. 2019;19:9.

\section{Publisher's Note}

Springer Nature remains neutral with regard to jurisdictional claims in published maps and institutional affiliations.

Ready to submit your research? Choose BMC and benefit from:

- fast, convenient online submission

- thorough peer review by experienced researchers in your field

- rapid publication on acceptance

- support for research data, including large and complex data types

- gold Open Access which fosters wider collaboration and increased citations

- maximum visibility for your research: over 100M website views per year

At BMC, research is always in progress.

Learn more biomedcentral.com/submissions 